\title{
An Analysis of Rheumatoid Arthritis Hospitalizations
}

Sandhya Shri Kannayiram ${ }^{1}$, Armaan Guraya ${ }^{2}$, Chukwudi C. Muojieje ${ }^{3}$, Karun M. Nair ${ }^{1}$, Osahon N. Idolor ${ }^{4}$, Jesse Odion ${ }^{5}$, Osaigbokan P. Aihie ${ }^{6}$, Eseosa Sanwo ${ }^{4}$

1. Internal Medicine, John H. Stroger, Jr. Hospital of Cook County, Chicago, USA 2. Medicine, Midwestern University Chicago College of Osteopathic Medicine, Downers Grove, USA 3. Internal Medicine, Mountain View Regional Medical Center, Las Cruces, USA 4. Medicine, University of Benin College of Medicine, Benin City, NGA 5. Internal Medicine, University of Benin Teaching Hospital, Benin City, NGA 6. Medicine, School of Medicine, University of Missouri, Columbia, USA

Corresponding author: Sandhya Shri Kannayiram, sandhya.kannayiram@yahoo.com

\section{Abstract \\ Background}

We used a large United States (US) population-based database to analyze the reasons for hospitalization of rheumatoid arthritis (RA) patients.

\section{Methods}

The International Classification of Diseases, Tenth Revision (ICD-10) code was used to search for hospitalizations in 2017 in the National Inpatient Sample (NIS) database with RA as the principal or secondary diagnosis. The reasons for hospitalization were divided into 19 categories based on their principal discharge ICD-10 diagnosis code. We also ranked the five most common specific reasons for hospitalization.

\section{Results}

There were over 35 million discharges included in the 2017 NIS database; 565,440 hospitalizations had either a principal or secondary ICD-10 code for RA. The top five reasons for RA hospitalization by ICD-10 code categories were as follows: cardiovascular (CV): 93,825 (16.59\%), rheumatologic: 82,785 (14.64\%), respiratory: 66,895 (11.83\%), infection: 62,660 (11.09\%), and injury/poisoning: 56,460 (9.96\%). Sepsis was the most common principal diagnosis for RA hospitalizations.

\section{Conclusion}

$\mathrm{CV}$ diseases were the most common ICD category, and sepsis was the most common principal diagnosis for RA hospitalizations. Management of medical comorbidities (such as CV) and prevention of infection is essential for reducing the rates of RA hospitalizations.

Review began 12/16/2020 Review ended 12/19/2020 Published 12/28/2020

\section{() Copyright 2020}

Kannayiram et al. This is an open access article distributed under the terms of the Creative Commons Attribution License CC-BY 4.0., which permits unrestricted use, distribution, and reproduction in any medium, provided the original author and source are credited.
Categories: Internal Medicine, Rheumatology, Epidemiology/Public Health

Keywords: : rheumatoid arthritis, hospitalization, sepsis, infection, cardiovascular, mortality, national inpatient sample

\section{Introduction}

Rheumatoid arthritis (RA) is a chronic autoimmune disorder that mainly affects the synovial joints, affecting up to $0.24-1 \%$ of the global population. It is one of the most common inflammatory disorders, with a clear predilection towards the female gender, occurring at a ratio of 2.5:1 compared with men [1]. It manifests in varying frequencies across different countries and different ethnic groups, being more common in the United States (US) and northern Europe than Southern Europe and countries in Asia and Africa [2]. This reported difference in prevalence among different countries and ethnic groups has been attributed to genetic, environmental, and lifestyle factors as well as a lack of enough studies in developing countries. Although studies have shown that the incidence of RA is decreasing in countries with high prevalence, RA continues to be one of the major contributors to the global disease and disability burden [3,4].

RA has a significant impact on health-related quality of life, causing loss of functional capacity and disability in the long term [5]. It affects physical and mental well-being and is associated with an increased risk of poor sleep quality, anxiety, depression, and excessive fatigue [6]. Apart from affecting mental and physical health, RA also causes a substantial economic burden to the patients, their caregivers, hospitals, and society due to the loss of work capacity, poor quality of life, multiple comorbidities, and premature mortality associated with the disease [7].

RA, being a multisystemic inflammatory disease, is associated with extra-articular manifestations such as 
coronary artery disease, interstitial lung disease, anemia, stroke, peripheral vascular disease, and increased risk of thromboembolism, particularly with severity and longer duration of disease [8]. The disease by itself and the use of immunosuppressive agents for its treatment have caused an increased prevalence of serious comorbidities like osteoporosis, infections, renal disease, and lymphoproliferative disorders in patients with RA [9]. Severe RA is associated with decreased life expectancy, increased risk of hospitalizations, and a twofold increase in mortality [10]. Early diagnosis and recognition of RA and its comorbidities and a multimodality approach to treatment are essential to decrease the mortality rates associated with the disease.

Over the last decade, a few population-based studies have demonstrated decreasing mortality and hospitalizations among RA patients [11]. Furthermore, only limited national-level data is available in the US on the reasons for hospitalizations of RA patients. Hence, in this report, we used the National Inpatient Sample (NIS), a large US population-based database, to address this clinically relevant issue.

\section{Materials And Methods \\ Data source}

The NIS was used to search for hospitalizations in 2017 with the International Classification of Diseases, Tenth Revision (ICD-10) RA codes "M05" and "M06" as the principal or secondary diagnosis. NIS is the largest hospitalization-related database in the US [12-15]. It entails a $20 \%$ probability sampling of different strata, which is structured to be representative of hospitalizations at the national level [16-19]. Each hospitalization in the 2017 NIS has a principal diagnosis and up to 40 secondary diagnoses. The principal diagnosis is the main reason for hospitalization, while the remaining diagnoses are secondary diagnoses $[20,21]$. Since patient data in NIS is de-identified, and the NIS database is available to the public, this study was exempted from the institutional review board approval.

\section{Inclusion and exclusion criteria}

The study population consisted of all hospitalizations in 2017 with a principal or secondary RA ICD-10 diagnosis codes. We excluded hospitalizations for patients who were younger than 18 years. We used ICD-10 codes "M05" and "M06" to identify hospitalizations with a principal or secondary diagnosis of RA.

\section{Statistical analysis}

All statistical analyses were performed using the Stata software, version 16.

\section{Outcomes}

The total number of RA discharges, age, race, length of stay (LOS), and total hospital charges incurred were recorded. The reasons for hospitalization were divided into 19 categories based on their principal discharge ICD-10 diagnosis codes. We also ranked the five most common specific reasons for hospitalization. The principal diagnosis for hospitalization was considered to be the reason for hospitalization.

\section{Results}

There were over 35 million discharges included in the 2017 NIS database; 565,440 hospitalizations were for patients aged 18 years or above, who had either a principal or secondary ICD 10 code for RA. Patients were predominantly female (74\%), White (65\%), with an average age of 67 years. The average LOS was five days and the average total hospital charge was $\$ 57,418$. The top five reasons for hospitalization for adult RA patients by ICD 10 code categories in descending order of frequency were as follows (Table 1): cardiovascular (CV): 93,825 (16.59\%), rheumatologic: 82,785 (14.64\%), respiratory: 66,895 (11.83\%), infection: 62,660 (11.09\%), and injury/poisoning: 56,460 (9.96\%). Sepsis was the most common principal diagnosis followed by acute chronic obstructive pulmonary disease (COPD) exacerbation, pneumonia, osteoarthritis of the right knee, acute kidney injury (AKI) in descending order of frequency. RA was the 25th most common principal discharge diagnosis. 


\section{Cureus}

ICD-10 Code Admission Category

Certain infections and parasitic diseases

Neoplasms and diseases of the blood and blood-forming organs and certain disorders involving the immune mechanism

Endocrine, nutritional, and metabolic diseases

Mental, behavioral, and neurodevelopmental disorders

Diseases of the nervous system

13,820

Diseases of the eye and adnexa and ear

Diseases of the circulatory system

Diseases of the respiratory system

Diseases of the digestive system

Diseases of the skin and subcutaneous tissue

Diseases of the musculoskeletal system and connective tissue

Diseases of the genitourinary system

28,930

Pregnancy, childbirth, and puerperium

4,875

Certain conditions originating in the perinatal period

0

Congenital malformations, deformations, and chromosomal abnormalities

\section{TABLE 1: ICD-10 code categories for hospitalizations of rheumatoid arthritis patients}

\section{Discussion}

In order to shed light on the morbidity rate of patients with RA on a national level, we conducted a comprehensive review of the NIS database. The major findings of our study are as follows: i) the most common reason for hospitalization for adult RA patients as per ICD-10 code categories was CV, with sepsis as the most common principal diagnosis, and ii) rheumatologic disorders were the second most common ICD category for hospitalization; however, RA was the 25 th most common principal diagnosis for hospitalization.

We found CV diseases to be the most common cause of hospitalization in RA patients, which is in line with other studies [22,23]. This finding is consistent with what is known about the increased CV burden associated with RA in the absence of other risk factors. Nonetheless, this further emphasizes the need to optimize preventative CV practices in a patient population that is commonly overlooked as being high risk for $\mathrm{CV}$ diseases. Proper management in the primary care setting may prevent a sizeable number of hospitalizations among RA patients. Further studies are needed to clarify how aggressive an approach is required and whether prophylactic pharmaceutical intervention is warranted. There is prudence in the early collaboration between the rheumatologist and cardiologist.

Infection was the fourth most common ICD-10 code category; however, sepsis was the most common specific principal diagnosis for RA hospitalizations. The Institute of Rheumatology, Rheumatoid Arthritis (IORRA) cohort was found to have infections as the most common cause of hospitalized comorbidity, while another single-center study found infections to be the leading cause of death in RA patients admitted to their ICU $[24,25]$. Similarly, Other studies have found RA to be an independent risk factor for long-term mortality due to ICU admission with sepsis [26]. These findings speak to the importance of early detection of infections that can lead to sepsis because its existence leads to dire consequences in all patients. While complications of the aforementioned CV disease are of a more chronic nature, infectious sequelae require acute detection 
and remediation. RA patients may need a lower threshold of suspicion given that they may be on immunosuppressive therapies and should be identified and treated early on.

Rheumatologic disorders were the second most common ICD category for hospitalization; however, RA was the 25th most common principal diagnosis for hospitalization. This discrepancy underlines the utility in addressing the many comorbidities of RA: the disease may not drive hospitalizations, but patients' susceptibility to other diseases, both rheumatological and not, do. A multidisciplinary approach is necessary to reduce rates of hospitalization, and this may include patient education, as a study at a tertiary care hospital found only $1.5 \%$ of RA patients are "aware" of their disease [27]. The relationship between patient and physician must be optimized by providing patients with the tools of pharmacotherapy, lifestyle changes, and education, and by empowering the physician by raising their clinical suspicion for the detection and prevention of RA comorbidities.

The large sample size and nature of the NIS, which enabled us to categorize reasons for hospitalizations into different ICD-10 organ-system-based categories and specific reasons are some of the strengths of this study. However, there are some limitations to our study: i) the NIS is an administrative database that uses claims data via ICD-10 codes to characterize diagnoses, and hence there is a possibility of errors being associated with coding [28]; ii) this report reflects data on RA hospitalizations rather than on individual patients [29]; iii) most ICD-10 codes do not grade disease severity, and NIS does not contain information on medication compliance [30]. Hence, we could not determine if underlying RA severity, and immunosuppressant compliance may have affected the number of RA hospitalizations.

\section{Conclusions}

For adult patients with a history of RA, CV diseases were the most common ICD category for hospitalization, while sepsis was the most common principal diagnosis for hospitalization. Rheumatologic disorders were the second most common ICD category for hospitalization; however, RA was the 25th most common principal diagnosis for hospitalization. Though RA patients are likely to be admitted because of other rheumatologic disorders, they are far less likely to be admitted principally because of RA. Hence adequate management of medical comorbidities (e.g., CV and respiratory disorders) is needed to reduce the rate of hospitalization.

\section{Additional Information \\ Disclosures}

Human subjects: All authors have confirmed that this study did not involve human participants or tissue. Animal subjects: All authors have confirmed that this study did not involve animal subjects or tissue. Conflicts of interest: In compliance with the ICMJE uniform disclosure form, all authors declare the following: Payment/services info: All authors have declared that no financial support was received from any organization for the submitted work. Financial relationships: All authors have declared that they have no financial relationships at present or within the previous three years with any organizations that might have an interest in the submitted work. Other relationships: All authors have declared that there are no other relationships or activities that could appear to have influenced the submitted work.

\section{References}

1. Lee DM, Weinblatt ME: Rheumatoid arthritis. Lancet. 2001, 358:903-911. 10.1016/S0140-6736(01)06075-5

2. Silman AJ, Pearson JE: Epidemiology and genetics of rheumatoid arthritis . Arthritis Res. 2002, 4:S265-S272. 10.1186/ar578

3. Alamanos Y, Voulgari PV, Drosos AA: Incidence and prevalence of rheumatoid arthritis, based on the 1987 American College of Rheumatology criteria: a systematic review. Semin Arthritis Rheum. 2006, 36:182-188. 10.1016/j.semarthrit.2006.08.006

4. Cross M, Smith E, Hoy D, et al.: The global burden of rheumatoid arthritis: estimates from the global burden of disease 2010 study. Ann Rheum Dis. 2014, 73:1316-1322. 10.1136/annrheumdis-2013-204627

5. Rosa-Gonçalves D, Bernardes M, Costa L: Quality of life and functional capacity in patients with rheumatoid arthritis - cross-sectional study. Reumatol Clin. 2018, 14:360-366. 10.1016/j.reuma.2017.03.002

6. Lin MC, Guo HR, Lu MC, Livneh H, Lai NS, Tsai TY: Increased risk of depression in patients with rheumatoid arthritis: a seven-year population-based cohort study. Clinics (Sao Paulo). 2015, 70:91-96. 10.6061/clinics/2015(02)04

7. Birnbaum H, Pike C, Kaufman R, Marynchenko M, Kidolezi Y, Cifaldi M: Societal cost of rheumatoid arthritis patients in the US. Curr Med Res Opin. 2010, 26:77-90. 10.1185/03007990903422307

8. Spagnolo P, Lee JS, Sverzellati N, Rossi G, Cottin V: The lung in rheumatoid arthritis: focus on interstitial lung disease. Arthritis Rheumatol. 2018, 70:1544-1554. 10.1002/art.40574

9. Listing J, Gerhold K, Zink A: The risk of infections associated with rheumatoid arthritis, with its comorbidity and treatment. Rheumatology (Oxford). 2013, 52:53-61. 10.1093/rheumatology/kes305

10. Naz SM, Symmons DP: Mortality in established rheumatoid arthritis. Best Pract Res Clin Rheumatol. 2007, 21:871-883. 10.1016/j.berh.2007.05.003

11. Dadoun S, Zeboulon-Ktorza N, Combescure C, Elhai M, Rozenberg S, Gossec L, Fautrel B: Mortality in rheumatoid arthritis over the last fifty years: systematic review and meta-analysis. Joint Bone Spine. 2013, 80:29-33. 10.1016/j.jbspin.2012.02.005

12. Edigin E, Prado V, Salazar M, Shaka H: Lung involvement in systemic lupus erythematosus increases 
inpatient mortality: analysis of the National Inpatient Sample. Chest. 2020, 158:A1871. 10.1016/j.chest.2020.08.1623

13. Shaka H, Raghavan S, Edigin E: Patients with type 1 diabetes mellitus do not have worse outcomes compared to type 2 following admission for bacterial pneumonia. Chest. 2020, 158:A306. 10.1016/j.chest.2020.08.305

14. Shaka H, Akuna E, Asemota I, Edigin E, Eseaton PO, Nwachukwu P, Pavon MR: Abstract 16236: hyperthyroidism does not negatively impact the outcomes of patients admitted for atrial fibrillation: analysis of the National Inpatient Sample. Circulation. 2020, 142:16236-16236. 10.1161/circ.142.suppl_3.16236

15. Shaka H, Edigin E, Gomez TMA: Obesity paradox in outcome of patients admitted with atrial fibrillation/flutter. Am Heart J. 2020, 229:159. 10.1016/j.ahj.2020.10.008

16. Shaka H, Raghavan S, Edigin E: Is the presence of diabetes mellitus a poor prognostic factor during hospitalizations for bacterial pneumonia? An analysis of the Nationwide Inpatient Sample. Chest. 2020, 158:A334. 10.1016/j.chest.2020.08.331

17. Ojemolon PE, Shaka H, Edigin E, et al.: Impact of diabetes mellitus on outcomes of patients with knee osteoarthritis who underwent knee arthroplasty: an analysis of the Nationwide Inpatient Sample. Cureus. 2020, 12:e8902. 10.7759/cureus.8902

18. Edigin E, Shaka H, Eseaton P, et al.: Rheumatoid arthritis is not associated with increased inpatient mortality in patients admitted for acute coronary syndrome. Cureus. 2020, 12:e9799. 10.7759/cureus.9799

19. Edigin E, Ojemolon PE, Eseaton PO, Shaka H, Akuna E, Asemota IR, Manadan A: Rheumatoid arthritis patients have better outcomes when hospitalized for ischemic stroke: analysis of the National Inpatient Sample (Epub ahead of print). J Clin Rheumatol. 2020, 10.1097/RHU.0000000000001563

20. Edigin E, Ojemolon PE, Eseaton PO, Shaka H, Akuna E, Asemota IR, Manadan A: Systemic sclerosis is associated with increased inpatient mortality in patients admitted for acute coronary syndrome: analysis of the National Inpatient Sample (Epub ahead of print). J Clin Rheumatol. 2020, 10.1097/RHU.0000000000001634

21. Shaka H, Edigin E, Gomez TMA, Eseaton PO: Diabetic ketoacidosis is one of the major reasons for hospitalizations among type 1 diabetics which led to inpatient mortality. Findings from the Nationwide Inpatient Sample. Am Heart J. 2020, 229:159-160. 10.1016/j.ahj.2020.10.010

22. Gil-Conesa M, Del-Moral-Luque JA, Gil-Prieto R, Gil-de-Miguel Á, Mazzuccheli-Esteban R, RodríguezCaravaca G: Hospitalization burden and comorbidities of patients with rheumatoid arthritis in Spain during the period 2002-2017. BMC Health Serv Res. 2020, 20:374. 10.1186/s12913-020-05243-0

23. Romano S, Salustri E, Ruscitti P, Carubbi F, Penco M, Giacomelli R: Cardiovascular and metabolic comorbidities in rheumatoid arthritis. Curr Rheumatol Rep. 2018, 20:81. 10.1007/s11926-018-0790-9

24. Sugimoto N, Nakajima A, Inoue E, et al.: Incidence of comprehensive hospitalization due to infection, cardiovascular disease, fractures, and malignancies in patients with rheumatoid arthritis. Rheumatol Int. 2017, 37:1871-1878. 10.1007/s00296-017-3811-5

25. Haviv-Yadid Y, Segal Y, Dagan A, et al.: Mortality of patients with rheumatoid arthritis requiring intensive care: a single-center retrospective study. Clin Rheumatol. 2019, 38:3015-3023. 10.1007/s10067-019-04651w

26. Barrett O, Abramovich E, Dreiher J, Novack V, Abu-Shakra M: Short- and long-term mortality due to sepsis in patients with rheumatoid arthritis. Rheumatol Int. 2017, 37:1021-1026. 10.1007/s00296-017-3694-5

27. Khalil Z, Salim B, Nasim A, Malik S: Patients' knowledge on rheumatoid arthritis - a study at a tertiary care hospital. J Pak Med Assoc. 2017, 67:256-260. https://pubmed.ncbi.nlm.nih.gov/28138181/

28. Edigin E, Eseaton P, Kaul S, et al.: Systemic sclerosis is not associated with worse outcomes of patients admitted for ischemic stroke: analysis of the National Inpatient Sample. Cureus. 2020, 12:e9155. 10.7759/cureus.9155

29. Edigin E, Kaul S, Eseaton PO: Analysis of hidradenitis suppurativa hospitalizations: a report from the National Inpatient Sample database (Epub ahead of print). J Am Acad Dermatol. 2020, 10.1016/j.jaad.2020.10.083

30. Edigin E, Ojemolon PE, Eseaton PO, Shaka H, Akuna E, Asemota IR, Manadan A: Systemic sclerosis is associated with increased inpatient mortality in patients admitted for atrial fibrillation: analysis of the National Inpatient Sample (Epub ahead of print). J Clin Rheumatol. 2020, 10.1097/RHU.0000000000001543 